\title{
12 \\ Conclusion: Thinking Is Good for You
}

The hope motivating these explorations was that thinking about the conceptual foundations of epidemiology could be good for both philosophy and epidemiology. These are only preliminary explorations, but initial appearances suggest that there are benefits to both disciplines. If epidemiology is good for you and thinking about epidemiology is good for epidemiology, then at least to this modest extent, thinking is good for you. Unfortunately for us, however, the thinker benefits only as a member of the population whose health epidemiology improves.

The common theme through much of this discussion has been the importance of explanation and the relative de-emphasis of causation. We have repeatedly seen that thinking about causation is less helpful than thinking about causal explanation. This is so because of the model of explanation we have focused on: contrastive causal explanation. The central plank of epidemiological method is to compare groups, in the hope that differences between them tell us something about the causes of their maladies. A contrastive condition on causal explanation requires that explanations cite not only cite causes but causes of differences. This is why explanation, as we have understood it, has proved such a useful conceptual tool with which to resolve traditional headaches about causation.

However, this short study covers only a part of what there is to be covered. The philosophy of epidemiology remains a fertile but largely unploughed field. We have set about achieving some specific goals: understanding the causal implications of epidemiological claims, modelling stable causal inference, modelling good prediction, resolving difficulties in the understanding of attributability, challenging risk relativism, questioning multifactorial thinking about disease, and clarifying the proper use of epidemiological evidence in certain legal settings. The larger goal, however, is to demonstrate that epidemiology rewards philosophical study, in the hope that others will undertake it and do it better. 